\title{
Use of Informational/Instructional Sheets with Eczema Patients
}

\author{
Craig G. Burkhart ${ }^{*}, 1$ and Craig N. Burkhart ${ }^{2}$ \\ ${ }^{1}$ University of Toledo College of Medicine, USA \\ ${ }^{2}$ University of North Carolina at Chapel Hill, Chapel Hill, North Carolina, USA
}

\begin{abstract}
Contact dermatitis is not a homogenous entity, but rather a number of subtypes contributing to different clinical presentations. For better understanding, instructional sheets provide information that assists in patient care. Informational sheets on contact dermatitis, patch testing, hand dermatitis, stasis dermatitis, and poison ivy are reviewed for open forum. Although often considered archaic therapy, restriction in use of soaps/cleansers appears to aid in clinical improvement of contact irritant dermatitis. Patient education on patch testing is greatly aided by presenting visual material prior to formal discussion. An internet site for more information regarding culprit environmental allergens is presented. Some simple methods for patients to achieve clinical improvement with eczema, hand dermatitis, and poison ivy are presented.
\end{abstract}

Simplicity, clarity, as well as practical, usable information are important with patient presentation of the subject of eczema. The material has to be presented in an easy-tofollow, coherent manner for patients. Semantics and scientific detail are not necessarily included. Thus on instructional sheets,

- $\quad$ eczema can be equated with dermatitis;

- determining the primary lesion in an eruption is not discussed;

- $\quad$ classification by stage, location, appearance, or cause is not necessary.

Eczema is inflammation of the upper layers of the skin. There is a range of skin rashes associated with the condition including redness, skin edema, dryness, crusting, flaking, blistering, cracking, oozing, and/or bleeding. The condition can be associated with temporary skin discoloration, but scarring is rare.

\section{CONTACT DERMATITIS}

The general handout for this entity (informational sheet \#1) categorizes dermatitis into contact irritant and contact allergic dermatitis. Irritant contact dermatitis is not a homogenous entity, but rather a number of subtypes contributing to different clinical presentations. The sheet provides suggestions in terms of skin care to the inflamed skin. Of note, a reduction in the use of soap to affected areas is strongly advised. Although often considered archaic therapy, a recent study strongly supports that soap/cleanser avoidance greatly assists patients with contact dermatitis [1].

In addition to the sheet, parents of neonates and young children are routinely told that young children's skin has fewer skin layers, and as a result, does not retain moisture as well. Thus, dryness can develop more easily in this age group. In older age groups, the message is that their oil

*Address correspondence to this author at the University of Toledo College of Medicine, 5600 Monroe Street, Suite 106B, Sylvania, Ohio 43560, USA; Tel: 419-885-3403; Fax: 419-885-3401; E-mail: cgbakb@aol.com glands secrete less, and dryness can be seen again. Thus, bathing habits which may have been performed for years, may now cause problems given this change in body function.

A new method for assessing irritation and dryness potential of soaps and washes has been the forearm-controlled application test. A number of instrumentally assessed measures, such as transepidermal water loss and skin hydration, can be used with this method to profile the skin-damaging potential of products. The soaps that listed on the informational sheet have proven to be the mildest by such irritation studies [2].

There remains confusion with atopic dermatitis and contact irritant dermatitis. As presently presented by textbooks, atopic dermatitis is a common chronic relapsing disease. It is multifactorial, heterogeneous and secondary to the interaction of many genes with environmental factors. It was initially categorized as a prurigo-like condition [3]. In the late $1890 \mathrm{~s}$ it was termed 'disseminated neurodermatitis' to emphasize that pruritus preceded the rash [4]. In the 1930s, dermatologist favored avoiding the issue of a psychiatric basis for the disease, and drew an association with atopy, asthma, and hay fever, leading to its present designation [5].

Of note there are no agreed diagnostic criteria for atopic dermatitis, and there is no consensus about its definition. Contact irritant dermatitis, on the other hand, has been defined by its four main component, namely skin barrier disruption, epidermal skin changes, cytokine release, and nerveending changes $[6,7]$. The wide discrepancy in defining manifestations of atopic dermatitis is reflected by the incidence and prevalence of 'atopic dermatitis' in the general population being reported to be as low as $0.8 \%$ in the Netherlands [8], 17\% in England [9] and 26.1\% in Nigeria [10]. Moreover, the reported prevalence rates in many developed countries dramatically increased in the early 2000 s, coinciding with the introduction of topical immunomodulators onto the market.

As classically described, atopic dermatitis should have a primary immunological abnormality of atopy, namely and elevated production of allergen-specific IgE. This criterion is 
rarely met. Thus, the condition may be better called 'atopiform contact irritant dermatitis'or basically just one subtype of contact irritant dermatitis [11].

Major facets in the management of contact dermatitis are prevention and therapeutic measures. Preventive measures include the use of moisturizers and reduction in bathing. With washing, one is dealing with patient habits, which are not easily altered unless discussed fully. Treatment for contact dermatitis includes topical and systemic corticosteroids, antibiotics, antihistamines, immunomodulating agents, and topical agents which are numbing, cooling, with anesthetic effects $[6,11]$.

Bathing is arguably the most important, but least studied, issue among such patients. The stratum corneum is structured with multi-lamellar lipid layers sandwiched between layers of flat, overlapping, and interlocking proteinaceous keratinocytes. The optimal level of hydration is maintained by regulating levels of natural moisturizing factor [12]. However, natural moisturizing factor and skin defensins (effector molecules of the cutaneous defense system) are soluble and leached from the stratum corneum structure with soap and water $[12,13]$. Lipids are lost by micelle formation with soap molecules [14]. Additionally, cleansers are formulated with surfactants which damage surfactants and solubilize lipids [15]. Indeed, the normal condition of the outer skin layer is largely determined by one's cleansing routines, such as the products used, and the frequency and context of usage [12].

There are many proponents of this therapeutic approach of limiting soap usage in dermatitis. Decades ago, most of the leading dermatologists advised that baths should not be given to eczematous infants and they were to be washed with tepid water [16-20]. In the 1960s the Scholtz regimen again prohibited bathing in eczematous patients [21, 22]. Even today, two Toledo hospitals have eliminated diaper wipes and only allow wetted gauze pads for diaper changes in newborn babies. Also, elimination of soap is routinely suggested for contact irritant dermatitis in some countries, including China [23].

Daily bathing has become routine for many in developed countries serving different hygienic, cosmetic, medical, social, cultural, and religious functions. And yet in reflecting back, hot water wasn't in homes till the 1930s and even into the 60s many people took baths only on Saturdays.

Besides suggesting a change in cleansing habits, today's patients are known not to comply with physician suggestions in general. For example, in one study $24 \%$ of patients did not even fill prescriptions of topical steroids for contact dermatitis [6].

Exposure to soap and cleansers (including super-fatted soaps, bath oils, and syndets) have long been recognized in laboratory studies to be an exacerbating environmental factor causing contact dermatitis. Nevertheless, the frequency of personal washing has substantially increased with soap and detergent personal wash products increasing seven-fold over the last 20 years [24]. The detrimental effects of soap arise through damage to the lipid lamellae, increase in skin $\mathrm{pH}$ (enhancing the activity of skin proteases), and damage to the barrier-related parameters. A single washing of the skin causes delipidation [25] and dehydration of the stratum cor- neum, with a shift of the $\mathrm{pH}$ of the skin's outer layer into the alkaline range, and ensuing irritation and impairment of the epidermal permeability barrier function [26]. It takes 12 hours for physiological function of the skin to be restored [27]. Additionally repeated washings with soap further reduce the normal skin flora, leading to increased colonization of the skin with more pathogenic organisms. For example, hand washing with soap increases bacterial counts [28].

In our media-driven culture, patients are often surprised that over washing could be causing any medical problems. Soaps do create a feeling of cleanliness by removing soils from the body and leaving a fresh scent. However, the public is not being told that one must balance hygienic effect with the skin damage which soap causes. The public does not realize that their normal skin condition is largely determined by their cleansing routines - the products they use and the frequency of use [12]. They don't associate their dry skin with their cleansing routines. At times it seems that companies are interested in drying people's skin, so that moisturizers and a host of other skin products become necessary. For example, there are no established criteria for mildness of cleansing products and several products designated for sensitive skin can demonstrate considerable irritant effects when tested $[29,30]$. Very often physicians have no more knowledge than their patients as to which soaps to recommend to patients with contact dermatitis. A change in attitude towards exposure to soap and other irritants and use of personal skin protection can only be achieved if the medical community provides them with how and why to use particular products.

Contact dermatitis is a common condition that can be induced or worsened by skin cleansing. Besides topical corticosteroids to reduce cutaneous inflammation, physicians have to be more adept at aiding patients in cleanser system selection. This includes for some individuals, greatly reducing bathing frequency and use of the appropriate cleansers and moisturizers. Fundamental skin care recommendations have to be addressed by physician groups, as the market place has its own interests at heart.

As noted on the instructional sheet, topical steroids are routinely given for the condition. On point, several retailers in America will dispense an $80 \mathrm{gm}$ tube of $0.025 \%$ triamcinolone, $80 \mathrm{gm}$ tube of $0.10 \%$ triamcinolone ointment, or 2 ounces of fluocinolone acetate solution for $\$ 4$ (informational sheet \#2). As an aside unrelated to the topic on hand, one can also purchase 30 pills of terbinafine, 20 tablets of $500 \mathrm{mg}$ of ciprofloxacin, or 20 tablets of doxycycline for the same, $\$ 4$.

\section{CONTACT ALLERGIC DERMATITIS}

Contact allergic dermatitis is an eczematous dermatitis caused by exposure to substances in the environment. Some contact allergies have characteristic distribution pattern and the cause can be most suggestive by history and clinical presentation. In such case, elimination of the suspected offending agent and appropriate treatment for eczematous inflammation should effectively solve the patient's problem. When an allergic dermatitis is suspected but the exact culprit is not apparent patch testing can prove most helpful. One standard patch test series in America is the Allergen Thinlayer Rapid Use Epicutaneous (T.R.U.E). Test. Informational sheet \#3 details patients several important points regarding patch testing. 
Once the culprit environmental allergens are determined, informational sheets should be available for patient on the topic. One source of information is Allergen's web page http://www.truetest.com/commonallergens.aspx, which offers specific information concerning where an allergen is found, how to avoid it, where one looks to avoid the allergen, what are some of the products that may contain the allergen, and what are some similar products which do not contain the allergen. One may offer additional suggestions to patients. For example, with nickel sensitive patients, I have added the following information:

\begin{abstract}
The palms of the hands have thick skin and do not develop a nickel allergic reaction. Thus, you can touch nickel with your hands all you want. However after touching something metallic (like a spoon, a metal pen, a cell phone, etc.), one can not touch one's arms, scratch the face, or rub your tummy. You will be transferring that metal to other body areas. Therefore, you have to get used to not touching your skin with your hands at all. If you have to scratch another body part, use some wooden body scratcher or a handkerchief.
\end{abstract}

\section{HAND DERMATITIS}

A separate instructional sheet (\#4) is presented because inflammation of the hands is one of the most common problems encountered by dermatologists. Dermatitis in this area causes discomfort, embarrassment, and interferes with normal daily activities. The most common type of hand inflammation is irritant hand dermatitis. The treatment advise for this condition is also apropos for other forms of hand dermatitis as well.

Frequent hand washing not only increases skin damage, but also can actually increase the number of microorganisms that colonize the skin [31]. In a different study, hand washing of damaged skin is not effective in reducing their contamination with epidemiologically important microorganisms [32].

\section{POISON IVY DERMATITIS}

The last informational sheet applies to rhus dermatitis (informational sheet \#5). In the United States, poison ivy, poison oak, and poison sumac are reported to produce more cases of allergic dermatitis than all other allergens combined. The eruption occurs from contact with the leaf or internal parts of the stem or roots and can be acquired during the winter months from the roots and stems. The clinical presentation varies with the quantity of sap that contacts the skin, how long it was on the skin, the pattern in which contact was made, individual susceptibility, and regional variations in cutaneous reactivity.

Linear lesions caused by when the plant is drawn across the skin or from streaking the oleoresin which scratching is very characteristic. Unusual patterns can be seen when the sap is acquired from contaminated animal hair, or clothing, or from smoke from burning plants. Given individual immunological differences to the allergen, the eruption may appear as quickly as 8 hours after exposure or may be delayed for an entire week. The appearance of new lesions a week after contact is confusing to patients, who mistakenly attribute the lesions to spread by scratching or via blister fluid. Blister fluid does not contain oleoresin and cannot spread the rash.

\section{STASIS DERMATITIS}

Stasis dermatitis is not a primary skin disease. It represents the cutaneous manifestation of a systemic illness, namely venous insufficiency. The condition is associated with incompetence of valves in the perforating veins of the leg. Venous hypertension, that develops, is associated with obliteration and thrombosis of venules and nutrient vessels.

The epidermal inflammation of stasis dermatitis can be managed similarly to contact irritant dermatitis with the instructional sheet discussed above. Of note, ointments are preferred because of the tendency of these patients to develop contact sensitization or irritation to various ingredients, such as preservatives and perfumes.

In an effort to address the underlying venous insufficiency, some guidelines (instructional sheet \#7) are helpful. By means of this sheet, one can underline the importance of hemodynamic support, supportive stockings, and leg elevation, as well as suggesting follow-up for that portion of the problem with the primary care physician.

The broad arena of eczema is not a homogenous entity, but rather a number of subtypes contributing to different clinical presentations. For better understanding, instructional sheets provide information that assists in patient care. Several statements made in this article are not evidence-based, and therefore, somewhat controversial. Nevertheless, a template for informational sheets on contact dermatitis, patch testing, hand dermatitis, stasis dermatitis, and poison ivy are presented for open discussion.

\section{Craig G. Burkhart, MPH, MD, Dermatologist}

5600 Monroe Street, Suite 106B, Sylvania, Ohio 43560, USA

\section{Information on Dermatitis}

Dermatitis, or eczema, means inflammation of the skin. This can be caused by a variety of internal and external factors. It may be due to an allergic reaction to a variety of agents (such as poison ivy, nickel, rubber) or due to an irritant reaction. Patients with irritant dermatitis are considered to have sensitive skin.

\section{General treatment recommendations include:}

I advise a dramatic reduction of washing with soap and cleansers to the area of the rash. Soap and cleansers further dries and irritates diseased skin, as well as remove the skin normal defenses (like defensin proteins). The use of hot water is also not advisable. For the first week of treatment, I would suggest no soap at all to the areas of rash. For example, if the rash is on the arm, leg, or neck, avoid all soap and cleansers to that area for a week. One can still shower, but don't put soap directly on the areas of dermatitis. I am old enough to remember the $50 \mathrm{~s}$ when $80 \%$ of people bathed once weekly (daily sponge bathing the armpits, groin, and scalp over the sink was preferred). One must learn to balance the hygienic effects of soap with the skin irritation which soaps cause. 
- $\quad$ Applying body lotion to the affected areas directly after drying off from showering.

- Low humidity, over-heated indoor environments further dry skin. The use of humidifiers or vaporizers is encouraged during the winter months.

- Chlorinated swimming pools, whirlpools, and hot tubs tend to dry the skin. One may need to apply emollients prior to (and after) these events in severely affected areas.

- $\quad$ Do not use fabric softeners that go into the dryer (i.e. Bounce). The particles within these softeners can irritate the skin in some individuals. Softeners that go into the wash (i.e. Downey, Yes, Bold) are recommended.

- $\quad$ It is also important to keep the area hydrated with moisturizers. Ointments are messy, but are the best moisturizer for fissures, such as cracked lips, and cracks on the palms and soles. Creams and gels are thicker than lotions and are preferred for most dermatitis rashes. Unless otherwise stated, I have no preference in specific over-the-counter brands in your selection of ointment, creams, or lotions.

- $\quad$ If used to affected skin, the soaps preferred are Dove Beauty Soap (white) Bar, Cetaphil Gentle Cleansing Bar, Dove Deep Moisture Beauty Wash, Cetaphil Gentle Skin Cleanser, or their generic equivalents such as Equate Skin Cleanser Gentle Formula (at Wal-Mart) and TopCare Ultra Moisturizing Body Wash (at Pharm). Examples of all of these products are in the office.

- Scratching can become a habit. There are books on behavioral modification that can be helpful if this is a problem in your case. An example would be Thomas Jefferson who suggested 'pinch oneself and count to 10 ' before considering scratching one's skin. A condition named lichen simplex chronicus is where the body has an electrical short and just keeps firing the words "Scratch Me." There are many beliefs, like yoga, which believe that you can control your body habits, including scratching, when one is asleep.

- Over-the-counter Lanacane and Sarna lotion can also help itchy skin. Another ingredient, pramoxine, also might alleviate the itch.

- $\quad$ There is no magical cure for dermatitis. Prescription non-fluorinated topical steroids for your condition are often used because of their high safety over prolonged usage. I often use prescription creams by day, and ointments at night.

\section{Craig G. Burkhart, MD, MPH}

5600 Monroe Street, Suite 106B, Sylvania, Ohio 43560, USA

\section{The \$4 Presciption Offerings at Several Retailers}

In an effort to make medications more affordable, I have reviewed the drug list on Wal-Mart's \$4 treatment program (Please note that there are also similar promotions at Pharm, Kroger, Meijer, etc). There are several drugs which are applicable to my patients. Of course with many prescription plans (such as mail-in forms), it may well be less expensive to continue receiving your medications in that manner (rather than the $\$ 4$ treatment plan). Below I list the drugs, by category, which can be substituted. Unfortunately, the bulk of the drugs we use are not on the list.

If you find that you can make use of the program, take your present prescription to Wal-Mart or the Pharm (or other store with the $\$ 4$ treatment program). Now my prescription might be written for more pills than allowed under the $\$ 4$ treatment program, but the pharmacist can modify the prescription so that you get the amount allowed under this new program. If the pharmacist appears confused, have him call my office staff and we can assist the pharmacist with any adjustment of your prescription.

\section{Antibiotics}

Amoxicillin

Cephalexin (also called Keflex)

Ciprofloxacin (also called Cipro)

Doxycycline (which could be a substitute for Minocycline)

Erythromycin

Penicillin VK

SMZ/TMP (also called Bactrim)

Tetracycline (which could be a substitute for Minocycline)

Fungal Treatments

Fluconazole (also called Diflucan)

Nystatin

Terbinafine (also called Lamisil)

Acne Treatment

Benzoyl peroxide $4 \%$ creamy wash

Topical Steroids

Hydrocortisone $2.5 \%$ cream

Fluocinolone acetate solution

Triamcinolone $.025 \%$ cream

Betamathasone valerate $0.1 \%$ ointment

\section{Viral Treatment}

Acyclovir (a generic form of Valtrex)

\section{Craig G. Burkhart, MPH, MD}

5600 Monroe Street, Suite 106B, Sylvania, Ohio 43560, USA

\section{Information on Patch Testing}

When allergic dermatitis (or allergic eczema) is suspected, patch testing is often the next step. Patch testing is a method for detecting contact allergies to specific chemicals that come in contact with the skin. These chemicals may be found in items such as topical lotions, creams, topical medications, gloves, shoes, clothing, jewelry, hair dyes, cosmetics, perfumes, and numerous other sources in the environment. My office uses the standard patch materials that test for the top 29 topical allergens in our environment excluding poison ivy. 
The chemicals to be tested have been already inserted into three long strips of paper, which I will apply to your back for 72 hours. I will fortify the adherent sheets with tape to secure its proper positioning. Thus, after 3 or 4 days you will need to return to the office so that I can read (interpret) the results of the patch testing.

Patch testing can be very helpful in determining the cause of many rashes, but sometimes no allergies are found. Although I may suspect that you are allergic to some external agent, patch testing may prove negative, meaning that you are not allergic to any of the items tested. In short, there are no guarantees that we will obtain any answers from this form of skin testing.

Patch testing is the best way to prove a contact allergic dermatitis, but it is not useful for other types of allergy. For example, the type of allergy that causes sneezing, sinus congestion, asthma, or hives is not detected by this method. Patch testing is not helpful to learn if you are allergic to items such as dog or cat dander, trees, grasses, flowers, foods, and the like.

Until you have returned to the office to have your patch tests read, you can only take sponge baths.

Until you have returned to the office to have your patch tests read, please try to avoid strenuous activities that would make you perspire enough to loosen the materials that are placed upon your back today.

If the sheets of materials that are taped to your back loosen, reinforce the strips to your back with tape from home.

Patch testing can cause itching especially if you develop a reaction to one of the chemicals tested. One can also get irritation from the tape used to fortify the materials to the back. One possible side effect is that one could develop temporary pigment changes on the back from a reaction to one of the chemicals tested.

\section{Craig G. Burkhart, MPH, MD}

5600 Monroe Street, Suite 106B, Sylvania, Ohio 43560, USA

\section{Information on Hand Dermatitis}

Hand dermatitis, or hand eczema, denotes inflammation of the skin either from irritant dermatitis (sensitive skin), or allergy (such as poison ivy, nickel, and rubber). Irritation is often from loss of God-given, normal protective oils and skin layers from repeated exposures to agents that remove these natural protective oils, such as soaps, detergents, and solvents. Although most people have 'tough' skin, some people have skin that is easily damaged. Skin protection becomes very important and is outlined below:

A. Wash your hands as few times as acceptable. Soap, by definition, removes oils from the skin. Don't listen to the advertisers, as no soap is "gentle to your skin." Go to any fast food restaurant and count how many people wash their hands before and after eating. The answer: not many. Try to follow their example until your condition improves. If you feel compelled to wash a lot, only wash your fingertips. If you wash your hands over 10 times a day, you will have problems regardless of any treatment given. If working in a factory, wipe the debris off your hands during day hours, and wash them with soap only on leaving to go home. On point, the skin makes defensin peptides that serve as our natural defense to bacteria, fungus, and injury. Washing these off with soap removes the Godgiven chemical shield against skin breakdown. By the way, several studies have shown that washing the hands does not decrease the number of bacteria at all. (Note: if some chemical gets on your hands that is caustic and burns, or if you work directly with human blood in a hospital setting, then, you would have to wash your hands on these two occasion).

B. Avoid jobs that require exposure to soapy water. Instead of washing dishes, buy plastic plates and utensils and throw them out. Also, eat out more often.

C. Wear waterproof, heavy-duty vinyl gloves when you are forced to do certain jobs with contact with soaps, detergents, scouring powders and the like. Paint and hardware stores have heavy-duty vinyl gloves for such purposes.

D. Wear waterproof gloves when peeling and squeezing lemons, oranges, or grapefruit, when pealing potatoes, and handling tomatoes.

E. Wear leather or heavy-duty fabric gloves when doing dry jobs and gardening. Also wear gloves outside to protect the hands from the cold in winter months.

F. Skin moisturizers are helpful. Apply emollients like hand lotion, or Vaseline intensive care lotion to the hands in between times of putting prescription agents to affected areas.

G. If one develops cracks, or fissures, on the hands, one must treat these similar to chapped lips. One avoids soap to these areas and applies ointments, like plain, old Vaseline. A daily application of greasy Vaseline with a Band-Aid to cracked skin is very helpful.

H. There is no fast magical cure for hand dermatitis. Your skin needs a rest from irritating agents. Unless you are careful with your hands, the dermatitis may recur.

I. Dr. Burkhart considers several different prescription treatments for this condition. Most of them are nonfluorinated topical steroids that can be used for prolonged time intervals (years) with high safety. Dr. Burkhart often suggests a topical cream to be used during the day hours, and an ointment base to the hands while sleeping.

\section{Craig G. Burkhart, MPH, MD}

5600 Monroe Street, Suite 106B, Sylvania, Ohio 43560, USA

\section{Information on Poison Ivy}

Three vine plants, namely poison ivy, poison oak, and poison sumac, cause poison ivy. In Toledo, poison ivy is by far the most common culprit. The plant's sap that flows through the leaves, stems, and roots is the allergen. Getting the rash of poison ivy means that you were exposed directly to the plant, or indirectly. Indirect exposure would be from touching someone's clothes who just worked outside (putting them into the wash for example), petting a dog who ran 
through the woods, or using a garden tool that previously was exposed to the poison ivy plant. Of note, poison ivy is not carried in the wind, except in the case of a forest fire. Also, although the plant grows much more in summer, one can get poison ivy in the winter months from the stem and roots of the plant.

Once exposed to the plant, one begins to break out 12 hours to 5 days later, depending upon one's immunologic response. After the first lesion appears, one breaks out with new lesions over the ensuing 2 to 3 weeks. Classically, one has streaks of blisters, which develop, on the arms and legs. The rash breaks out depending on how much got onto the skin, how long it was on before it was washed off with soap and water, and how sensitive the body parts are. The oozing from the blisters does not spread the rash (although this is a common belief).

The only reliable prevention is to recognize the plant and stay away from it. It is often stated to poison ivy sufferers, "Leaves of three, let it be." Shots and oral agents have attempted desensitization, but these have not proven to be effective therapies. Of note, there is an over-the-counter product, Ivy Block, which blocks the plant sap from getting onto the skin, as it acts like a sunscreen for sun protection.

The treatment of poison ivy is at two levels. The lower level is very safe, and consists of topical steroids and oral antihistamines. Unfortunately, for the very severe cases, this treatment may not give enough relief. However, if the face is not involved and you are able to sleep, I usually suggest this treatment. Indeed, this is the treatment I used when I had poison ivy.

The other treatment is oral (or injectable) steroids. Oral steroids get into the system in $1 / 2$ hour and are the safer route. One can not adjust the dose of injected medication, if side effects occur. As with all drugs, the benefits expected from the use of steroids must be weighed against potential side effects. Steroids add a stress to the system (the heart, the lungs, the whole body), and should be given with concern of its possible side effects. Of note, steroids usually have to be given for the duration of the possible breakout ( 2 or 3 weeks), or the rash may seem like its reflairing. I usually give enough steroids so that you feel more comfortable, but not necessarily enough to eliminate the entire rash (to reduce the possibility of side effects from steroids).

\section{Comments on Stasis Dermatitis from Craig G. Burkhart, MD, MPH}

Statis dermatitis refers to the skin changes that occur to the lower legs as a result of 'stasis' or blood pooling from insufficient venous return. Insufficient venous return results in increased pressure in the capillaries with the result that fluid and red blood cells leak out of the capillaries. Once red blood cells leak out of the vessels, they can't return to the vessels. During periods of sitting, standing, and walking, the blood in our leg veins must flow uphill to the heart. This is easy when we have healthy veins. It is aided by our calf muscles, which act as miniature pumping stations. With diseased veins, however, it can lead to venous congestion in our legs. This congestion can cause a number of problems such as varicose veins, swollen legs (edema), leg ulcers, and possibly thrombosis (blood clot). Additionally, the cracks and poor skin condition of this disorder predisposes one for entry of bacteria, and an infection referred to as cellulitis.

How can venous congestion in the legs be avoided? (1) By movement of the calf muscles (walking or moving the feet). The blood in your veins is pumped in the direction of your heart. This function is called the 'muscle pump'. (2) By keeping the legs elevated. It is far easier for your body to pump blood downhill than uphill. Elevating your legs eases the task of pumping blood from your legs to your heart. (3) By wearing medical elastic stockings, which, by external counter-pressure on varicose veins, speeds up the blood flow to the heart.

\section{Six easy steps to better blood flow:}

(1) Sitting and standing for long periods of time are bad for your body. It is better to lie down with your legs raised, or walk briskly when you can. This means more movement, walking, climbing stairs, cycling and swimming. It is helpful to do things that keep your leg muscles in motion.

(2) Activate your 'muscle pump'. When sitting and standing for long periods cannot be avoided, activate your 'muscle pump'. Move your feet up and down frequently with a 'pumping motion'. At work or during long trips by car, train, or plane, move your legs.

(3) Keep your feet higher than your heart. Take the load off your leg veins by frequently elevating your legs. During the day, prop your feet up on a stool, your desk, or a windowsill. At night, raise the foot of your bed, if the bed frame is adjustable. If not, place cushions under the mattress to elevate it approximately six inches.

(4) Avoid excessive heat. No sunbathing of the legs. And never let your legs become sunburned. No hot baths.

(5) Fight obesity. Excessive weight is a burden on your heart, your arteries, and your veins. Quite simply: Eat the right food... and eat less.

(6) Apply your medical elastic stockings regularly each morning. Note that we said 'medical elastic stockings' for a reason. So-called 'support stocking' are generally inadequate support for you legs and veins. Medical elastic stockings are generally prescribed by your primary car physician after a thrombosis (clot), during pregnancy, when the legs are swollen, or when varicose veins are pronounced.

Note: if you still have significant swelling after following the advice above, you may want to discuss the use of water pills with your primary care physician. You may either be a candidate for water pills, or you may need adjustment of your dosage.

\section{REFERENCES}

[1] Burkhart CG. Clinical assessment by atopic dermatitis patients of response to reduced soap bathing: A pilot study. Int J Dermatol 2008; 47: 1216-7.

[2] Abbas S, Goldberg JW, Massaro M. Personal cleanser technology and clinical performance. Dermatol Ther 2004; 17: 35-42.

[3] Johnson WR (ed.). On Cutaneous Diseases. London: London Press, 1808.

[4] Von Gebra H. Dermatomycosis Diffusa Fexorum, in die Krankhaften Veranderungen der Haut und Ihrer Anhangsgebilde Mit 
Ihrer Beziehungen $\mathrm{Zu}$ der Krankheiten Des Gesamtoranismus. Braunschweig: Wreden, 1884.

[5] Hill LW. Evolution of atopic dermatitis. Arch Dermatol 1931; 32: 451-6.

[6] Burkhart CG. Contact irritant dermatitis and anti-pruritic agents: The need to address the itch. J Drugs Dermatol 2003; 2: 143-6.

[7] Smith HR, Basketter DA, McFadden JP. Irritant dermatitis, irritancy and its role in allergic dermatitis. Clin Exp Dermatol 2002; 27: $138-46$.

[8] Verboom P, Hakkaart-Van Roijen L, Sturkenboom M, et al. The cost of atopic dermatitis I the Netherlands: An international comparison. Br J Dermatol 2002; 147: 716-24.

[9] Bleiker TO, Shahidullah H, Duttone E, Graham-Brown RAC. The prevalence and incidence of atopic dermatitis in a birth cohort: The importance of a family history of atopy. Arch Dermatol 2000; 136; 274-9.

[10] Falade AG, Olawuyi F, Osinusi K, Onadeko BO. Prevalence and severity of symptoms of asthma, allergic rhinoconjunctivitis and atopic eczema in secondary school children in Ibada, Nigeria. East Afr Med J 1998; 75: 695-8.

[11] Burkhart CN, Burkhart CG. Pilot study of patient satisfaction with nonfluorinated topical steroids compared with a topical immunomodulator in atopic dermatitis. Int J Dermatol 2004; 43: 215-9.

[12] Johnson AW. Overview: fundamental skin care -- protecting the barrier. Dermatol Ther 2004; 17: 1-5.

[13] Schroder JM, Glaser R, Harder J. Antimicrobial peptides: Effector molecules of the cutaneous defense system. Int Cong Ser 2007; 1302: 26-35.

[14] Burkhart CN, Kruge MA, Burkhart CG, Black C. Cerumen composition by flash pyrolysis-gas chromatography/mass spectrometry. Otolo Neurotol 2001; 22: 715-722.

[15] Polefka TG. Surfactant inteactions with skin. In: Zoller U, Broze G, Eds. Handbook of Detergents. Part A. Properties. New York, NY: Marcel Dekker, 1999: 433-68.

[16] Marfan AB. Clinique de la premiere enfance. Paris: Masson; 1928: 389-96.

[17] Babonneix L. Eczema du nourrison. In: Gougerot H, Ed. L'eczema, etiologie et pathogenie. Paris: Maloine 1935; 106-21.

[18] Duhring LA. Traite pratique des maladies de la peau, trad Barthelemy T et Colson A. Paris: G Masson 1883; 209.
[19] Bulkeley DL. Diet and hygiene in diseases of the skin. New York: Paul Hoeber; 1913.

[20] Sabouranud R. L'eczema du nourrisson. La Clinique 1906; 10: 147-8.

[21] Scholtz JR. Management of atopic dermatitis. Calif Med 1964; 100: 103-5.

[22] Scholtz JR. Management of atopic dermatitis. Calif Med 1965; 102: 210-6.

[23] Hon KLE, Leung TF, Wong Y, So HK, Li AM, Fok TF. A survey of bathing and showering practices in children with atopic eczema. Clin Dermatol 2005; 30: 351-4.

[24] Cork MJ, Robinson DA, Vasilopoulos Y, et al. New perspectives on epidermal barrier dysfunction in atopic dermatitis: geneenvironment interactions. Curr Rev Allergy Clin Immunol 2006; 118: 3-21.

[25] Fulmer AW, Kramer GJ. Stratum corneum lipid abnormalities in surfactant-induced dry scaly skin. J Invest Dermatol 1986; 86: 598602.

[26] Grunewald AM, Gloor M, Gehring W, Kleesz P. Damage to the skin by repetitive washing. Contact Dermatol 1995; 32: 225-32.

[27] Bornkessel A, Flach M, Arens-Corell M, Elsner P, Fluhr JW. Functional assessment of a washing emulsion for sensitive skin: mild impairment of stratum corneum hydration, $\mathrm{pH}$, barrier function, lipid content, integrity and cohesion in a controlled washing test. Skin Res Technol 2005; 11: 53-60.

[28] Chamberlain AN, Halablab MA, Gould DJ, Miles RJ. Distribution of bacteria on hands and the effectiveness of brief and thorough decontamination procedures using non-medicated soap. Zhl Bakt 1997; 285: 565-75.

[29] Loden M, Buraczewska I, Edlund F. The irritation potential and reservoir effect of mild soaps. Contact Dermatol 2003; 49: 91-6.

[30] Baranda L, Gonzalez-Amaro R, Torres-Alvarez B. Correlation between $\mathrm{pH}$ and irritant effect of cleansers marketed for dry skin. Int J Dermatol 2002; 41: 494-99.

[31] Winnefeld M, Richard MA, Drancourt M, Grob JJ. Skin tolerance and effectiveness of two hand decontamination procedures in everyday hospital use. Br J Dermatol 2000; 143: 546-50.

[32] Borges LFA, Silva BL, Filho PPG. Hand washing: Changes in skin flora. Am J Inf Control 2007; 35: 417-20.

(C) Burkhart and Burkhart; Licensee Bentham Open.

This is an open access article licensed under the terms of the Creative Commons Attribution Non-Commercial License (http://creativecommons.org/licenses/by$\mathrm{nc} / 3.0 /$ ) which permits unrestricted, non-commercial use, distribution and reproduction in any medium, provided the work is properly cited. 